This is an electronic reprint of the original article. This reprint may differ from the original in pagination and typographic detail.

Author(s): Kujala, Tuomo; Grahn, Hilkka; Mäkelä, Jakke; Lasch, Annegret

Title: On the Visual Distraction Effects of Audio-Visual Route Guidance

Year: $\quad 2016$

Version:

Please cite the original version:

Kujala, T., Grahn, H., Mäkelä, J., \& Lasch, A. (2016). On the Visual Distraction Effects of Audio-Visual Route Guidance. In P. Green, S. Boll, G. Burnett, J. Gabbard, \& S. Osswald (Eds.), AutomotiveUI 2016 : Proceedings of the 8th International Conference on Automotive User Interfaces and Interactive Vehicular Applications (pp. 169-176). Association for Computing Machinery. https://doi.org/10.1145/3003715.3005421

All material supplied via JYX is protected by copyright and other intellectual property rights, and duplication or sale of all or part of any of the repository collections is not permitted, except that material may be duplicated by you for your research use or educational purposes in electronic or print form. You must obtain permission for any other use. Electronic or print copies may not be offered, whether for sale or otherwise to anyone who is not an authorised user. 


\section{On the Visual Distraction Effects of Audio-Visual Route Guidance}

\author{
Tuomo Kujala \\ University of Jyväskylä \\ Jyväskylä, Finland \\ tuomo.kujala@jyu.fi
}

\author{
Hilkka Grahn \\ University of Jyväskylä \\ Jyväskylä, Finland \\ hilkka.grahn@jyu.fi
}

\author{
Jakke Mäkelä \\ University of Jyväskylä \\ Jyväskylä, Finland \\ jakke.makela@gmail.com
}

\author{
Annegret Lasch \\ Google \\ Munich, Germany \\ annegret.lasch@gmail.com
}

\begin{abstract}
This is the first controlled quantitative analysis on the visual distraction effects of audio-visual route guidance in simulated, but ecologically realistic driving scenarios with dynamic maneuvers and self-controlled speed $(N=24)$. The audio-visual route guidance system under testing passed the set verification criteria, which was based on drivers' preferred occlusion distances on the test routes. There were no significant effects of an upcoming maneuver instruction location (up, down) on the in-car display on any metric or on the experienced workload. The drivers' median occlusion distances correlated significantly with median incar glance distances. There was no correlation between drivers' median occlusion distance and intolerance of uncertainty but significant inverse correlations between occlusion distances and age as well as driving experience were found. The findings suggest that the visual distraction effects of audio-visual route guidance are low and provide general support for the proposed testing method.
\end{abstract}

\section{Author Keywords}

Driver distraction; navigation system; visual demand; visual occlusion; occlusion distance; intolerance of uncertainty.

\section{ACM Classification Keywords}

H.5.2. Information interfaces and presentation (e.g., $\mathrm{HCI}$ ): User Interfaces - Graphical user interfaces (GUI), Evaluation/methodology

\section{INTRODUCTION}

Widely used verification guidelines for visual-manual invehicle electronic devices of the National Highway Traffic Safety Administration (NHTSA) [9] recommend three metrics to verify the distraction potential caused by electronic devices (total and mean duration of in-car glances, percentage of over-2-second in-car glances). According to the guidelines, the testing scenario should consist of a straight highway driven with steady speed and keeping of a static distance $(70 \mathrm{~m})$ to a lead car.

Permission to make digital or hard copies of part or all of this work for personal or classroom use is granted without fee provided that copies are not made or distributed for profit or commercial advantage and that copies bear this notice and the full citation on the first page. Copyrights for third-party components of this work must be honored. For all other uses, contact the Owner/Author. Copyright is held by the owner/author(s).

Automotive'UI 16, October 24-26, 2016, Ann Arbor, MI, USA ACM 978-1-4503-4533-0/16/10

http://dx.doi.org/10.1145/3003715.3005421
However, real-world driving scenarios with route guidance include turns, lane selections, and the associated decelerating and accelerating behaviors, and thus, the distraction effects of navigation system cannot be reliably tested within the NHTSA scenario [6]. In real-world driving, visual route guidance is in fact realized typically in these types of dynamic situations when approaching turns or selecting lanes. Furthermore, the safety risk of a 2second in-car glance is highly dependent on the driving speed among other situational variables. The visual distraction effects of a 2-second off-road glance are significantly different while driving 60 than 80 kilometers per hour in a particular road environment [7].

The NHTSA verification guidelines [9] have received a lot of attention related to the effects of participant sampling on the outcomes of the testing (pass or fail, see e.g., $[1,3]$ ). Validation of relevant driver sample characteristics seem to be missing, and it has been argued that it depends mostly on the random driver sample if an in-car task passes or fails the criteria, that is, if the drivers happen to be 'short-glancers' or 'long-glancers'.

For the reasons above, the present study follows an alternative test environment and in-car glance duration verification criteria for dynamic and self-paced driving scenarios suggested by Kujala and Mäkelä $(2015$, [6]). This is the first time the proposed method is applied to a real incar task. The verification criteria are based on visual occlusion data mapped on the test routes.

The technique of visual occlusion is an established method to define the visual demands of driving [10,13]. In the technique, the visual field of the driver is intermittently occluded with an occlusion visor or opaque screens. The occlusion time (OT) and/or the occlusion distance (OD) the driver is able to drive without visual information of the forward roadway are measured. By the means of visual occlusion, the testing method presented in [6] utilizes the preferred occlusion distances of 97 drivers on simulated real Finnish roads [7] as a baseline (i.e., control) of acceptable in-car glances. Occlusion distance (OD) refers to the distance that a driver feels comfortable to drive with occluded vision while fully concentrating on the driving task [7]. Basically, the lower the occlusion distance for a road, the higher the environmental visual demands of the road. 
In the testing method of Kujala and Mäkelä [6], it is possible to define threshold ODs, which drivers are not willing to exceed while fully concentrating on the driving task, for each point of a route used for testing. Exceeding the threshold OD when looking at an in-car device indicates a failure in control of the visual sampling off road. In this situation, the driver is driving a longer distance without focal visual information of the road than the driver would be willing to drive occluded when fully focusing on the driving task.

A traffic light analogy is used by Kujala and Mäkelä [6] to define the acceptability of the individual in-car glances from the sample ODs. The specific threshold values are:

- Green: the median OD of the driver sample ODs for a given road point gives a value that is assumed to be still safe (drivers are assumed to have behaved cautiously on average).

- Red: the $85^{\text {th }}$ percentile OD gives a value that when exceeded, the glance can definitely be considered as unacceptable by the majority of the driver population.

A clear goal for an acceptable in-car task is to have all the glances within the green category but some tolerance must be accepted, as there are clear individual differences in the preferred occlusion distances [7]. Unfortunately, the authors [6] do not provide explicit verification criteria for acceptable percentages per glance category. For this reason, we took their original data $(N=97)$ and calculated what are the median percentages of occlusion distances below or at the median OD ('green occlusions') and occlusion distances exceeding the $85^{\text {th }}$ percentile OD ('red occlusions') of the drivers. Based on these percentages, the verification threshold of green glances was set to $68 \%$. That is, at least $68 \%$ of in-car glances should be shorter or at most at the median ODs (i.e., green). Accordingly, the verification threshold of red glances was set to $6 \%$. That is, at most $6 \%$ of in-car glances can be above the $85^{\text {th }}$ percentile ODs (i.e., red). In order for these thresholds to be representative, it is vital that the preferred occlusion distance distribution of a test sample is comparable to the original sample $(N=97)$, from which these threshold values are derived from.

The purpose of the current study was to run the first controlled quantitative analysis on the visual distraction effects of audio-visual route guidance in realistic driving scenarios with dynamic turns and self-controlled speed, and to further validate the test method of Kujala and Mäkelä [6]. The study is divided into two parts: distraction testing and occlusion experiment. The behaviors of the same participants were studied in the both parts of the study.

In the distraction testing we studied a commercial audiovisual route guidance prototype in a motion-platform driving simulator following the testing and verification criteria described in [6]. We investigated the visual distraction potential between two alternative navigation system display designs; maneuver box location up or down.
Maneuver box contains information about the next maneuver and the distance to the turn (see Figure 1).

The research questions for the distraction testing included:

1. Does the studied commercial prototype for route guidance pass the set verification criteria?

2. Are there significant differences in the visual distraction potential between the two alternative display designs (maneuver box up or down)?

3. Do the drivers experience more task workload with either of the two designs and how does the workload compare to the occlusion experiment (NASA-TLX; [4])?

4. Are the test results comparable on routes with different visual demands (suburban and highway)?

Based on previous research (for review, see [12]) and because crash statistics haven't shown significant effects of similar route finding tasks on crash risk, we hypothesized that the task to follow the audio-visual route guidance would pass the verification criteria regardless of the visual user interface design.

From a design point of view, the advantage of the maneuver box's lower position is that it allows to display also the following maneuvers in a natural position above the first one (Figure 1). However, we expected that the upper location of the maneuver box enables the driver to sample more efficiently the upcoming maneuver guidance and upcoming route on the map with a single glance, whereas larger movements of gaze are expected when the maneuver box is located farther apart down from the upcoming route. This could lead to longer individual in-car glance durations with the maneuver box down, if these two types of visual information are sampled within a glance.

For the method validation, the second part of the study was an occlusion experiment following [7]. The research questions were:

1. Is the OD distribution of our test sample comparable to the OD distribution for highway driving reported in [7] (the baseline data)?

2. Do the ODs correlate with the driver's preferred in-car glance distances (i.e., distance traveled during an in-car glance) across the in-car tasks?

3. Do the drivers' ODs correlate with their reported intolerance of uncertainty [2], age or driving experience? Intolerance of uncertainty is one plausible personality factor that could explain at least partly the individual differences in the preferred occlusion distances.

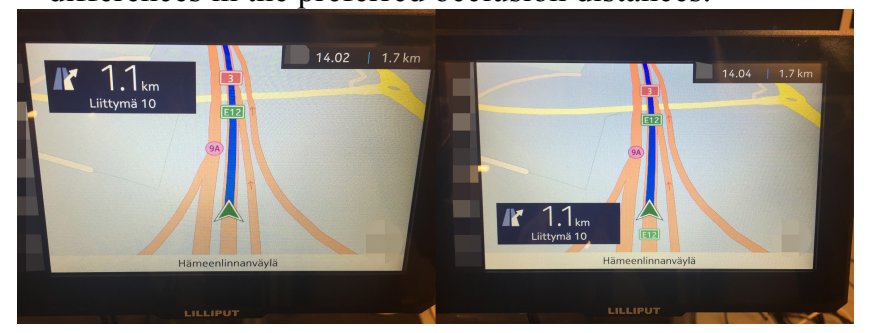

Figure 1: Maneuver boxes on the in-car display (left up, right down). 


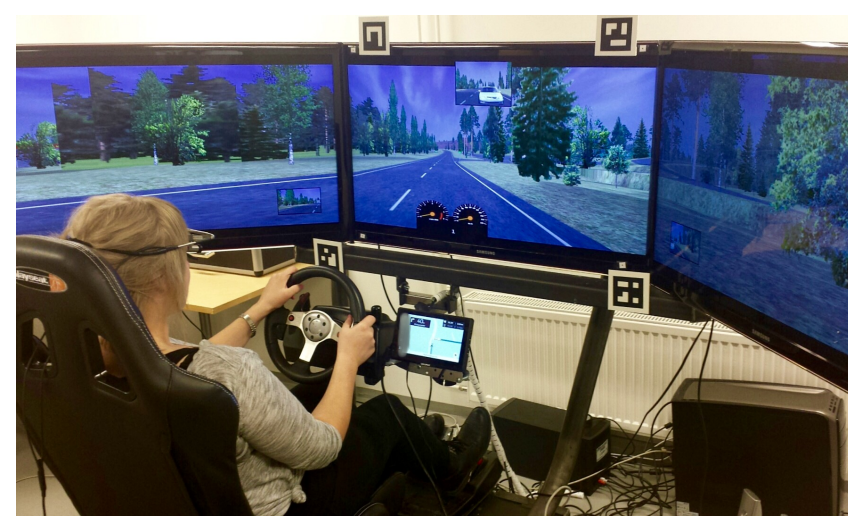

Figure 2: The experimental setup.

\section{METHOD}

The experimental design of the distraction test was withinsubjects $2 \times 2$. The independent variables were the maneuver box location (up, down, Figure 1) and the driving environment (highway, suburban). In the occlusion experiment the independent variables included age, driving experience, and the intolerance of uncertainty [2].

\section{Participants}

In total we had 24 participants who were recruited via email lists. We tried to follow the NHTSA [9] recommendations on the driver sample as closely as possible. Twelve of the participants were male and twelve were female. Participants' age varied from 21 to 67 years and mean age was $38,4(S D=15.3)$. Six of the participants were 18 to 24 years old, six 25 to 39 years old, eight 40 to 55 years old, and four over 55 years old. Half of the participants in each age group were male and half female. The age categories followed the NHTSA test participant recommendations [9]. The reason of the small deviation is because two older (55+) participants suffered from minor simulator sickness and were replaced with over 40 years old participants.

All participants had a valid driver's license and they drove at least 5,000 kilometers per year. The total distance driven varied from 5,000 to 30,000 kilometers $(M=13,300, S D=$ $6,900)$. The lifetime driving experience of the participants varied from four years to 49 years $(M=19.5, S D=14.5)$. All participants had normal or corrected-to-normal vision and were able to drive and navigate without glasses. Experiments were instructed in Finnish and all participants were fluent Finnish-speakers. All participants were unfamiliar with the prototype navigation system that was tested. Each participant was rewarded with a movie ticket and a car phone holder.

\section{Apparatus}

The experiments took place at the driving simulator laboratory at the University of Jyväskylä. The simulator can be categorized as medium-fidelity and it consisted of CKAS Mechatronics 2-DOF motion platform, longitudinally adjustable seat as well as Logitech G27 force-feedback steering wheel and pedals (Figure 2). Automatic transmission was used during the experiments. The driving scene was displayed on three 40" LED screens $(95.6 \mathrm{~cm} \mathrm{x}$ $57.4 \mathrm{~cm}$ ) and the resolution was $1440 \times 900$ pixels per screen. The middle screen included head-up display speedometer, RPM gauge and a rear-view mirror. Both side screens had side mirrors. For the occlusion experiment, the back of the steering wheel was equipped with a lever for removing the occlusion of the driving scene for 500 milliseconds for each press during the visual occlusion trial. Continuous pressing of the lever kept the driving scene

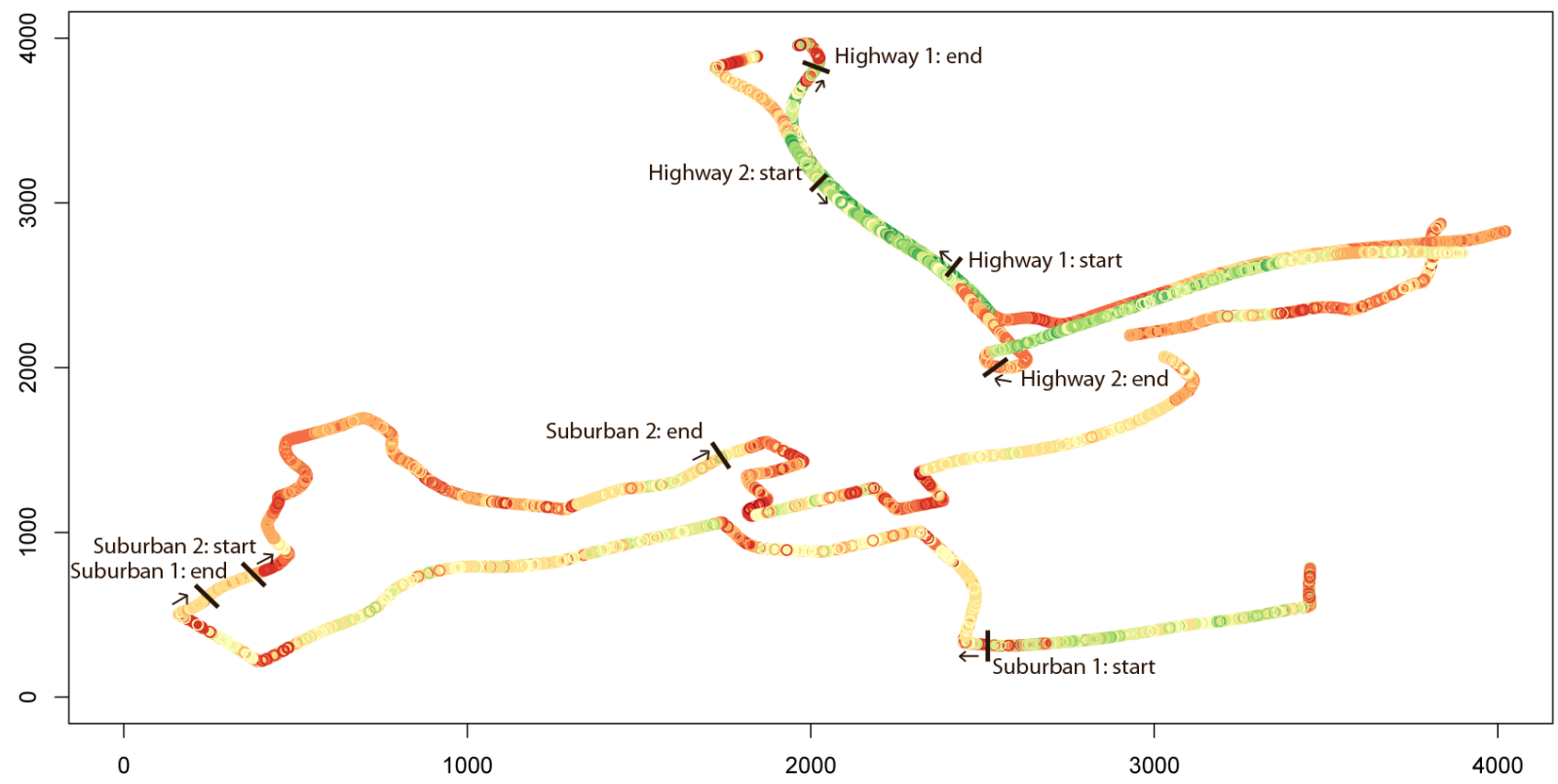

Figure 3: The pre-defined routes for the experiments. Green: low visual demands (high OD), red: high visual demands (low OD). NB. Roads are not in scale. 
continuously visible. The driving simulation software was provided by Eepsoft (http://www.eepsoft.fi/). The software saved driving log data at $10 \mathrm{~Hz}$. The used predefined routes simulated real Finnish highway and suburban roads located at Martinlaakso, Vantaa. The same roads were used as in the study of Kujala and Mäkelä [6] (see Figure 3). The driving simulator sent real-time simulated GPS data to the navigation system under testing to support route guidance.

The prototype navigation system was running on Intel NUC5i3RYK and displayed on Lilliput 779GL-70NP/C/T 7" capacitive touchscreen display. Ergoneers' Dikablis 50 $\mathrm{Hz}$ eye-tracking system was used to record participants' eye movements. LAN bridge was used for the synchronization of the driving simulator and eye-tracking data.

\section{Procedure}

After signing an informed consent, participants were taken to the driving simulator and the seat was adjusted for each participant. At first, participants practiced driving in an artificial city environment with other road users as long as they wanted. The average practice time was 3.2 minutes. After they felt comfortable with driving, they did a second practice on a suburban route in Martinlaakso with audiovisual route guidance on to get familiar with the guidance that the navigation system provided (two turns). When both practices were done, the eye-tracking headset was put on, adjusted and calibrated.

During the distraction testing, participants were instructed to follow the audio-visual route guidance to find the predefined destination. The participants were able to listen the route guidance as well as to see the route and the upcoming maneuvers displayed on a map on the touchscreen display. The routes were set by the experimenter, thus there was no manual input required from the driver during the trials. The participants were told to try to drive about 80 kilometers per hour on highway and about 50 kilometers per hour on suburban roads (speed limits), but that they can control the speed freely according to the situational demands. The routes were always driven in the same order, but counterbalancing was done for the design alternatives. In order to control the possible learning effects, there were two slightly different highway (one turn each; a ramp) and two slightly different suburban routes (five turns each), see Figure 3. The routes were selected by finding routes as similar as possible regarding their visual demands (for both route types). The order of the routes was counter-balanced across the sample. In total each completed four trials: two on a highway scenario $(1.4$ and $1.5 \mathrm{~km})$ and two on a suburban scenario $(3.7$ and $2.5 \mathrm{~km}$ ). There were no other road users in the scenarios. After each trial, NASA-TLX questionnaire [4] was filled out.

Fourteen out of 24 participants (58.3\%) got lost at least on one route. Most of the cases happened when the participant took a wrong turn just before the correct one. A possible reason for the high percentage could be the difficulty to assess distances in a driving simulator. The trials were rerun, unless the participant got lost in the last turn.

After the distraction testing, the visual occlusion experiment started. At first, the participants practiced in a city environment with other road users how to drive with vision occasionally occluded and how to use the lever that removed the occlusion of the driving scene. After the practice, the actual occlusion trial started. The average practice time was 3.3 minutes.

In the occlusion experiment, the screens were blank as default. By pressing the lever on the back of the steering wheel, the participant could remove the occlusion of the driving scene for 500 milliseconds at a time, following the original method by Senders et al. [10]. The findings of Senders et al. as well as Kujala et al. [7] suggest that 500 milliseconds of intermittent visibility is enough for at least experienced drivers to drive fairly fluently and according to traffic regulations in the studied scenarios.

The participants were instructed to follow the traffic regulations and drive safely but within these limits, try to drive vision occluded as long as possible. They were also told that six test participants who drive most accurately and the longest periods with occluded vision, get a second movie ticket. This was done in order to make the participants focus on the driving task but still try to maximize the preferred occlusion distance. The visual occlusion trial included highway routes only without other road users. The same highway routes were used as in the distraction test. The speed limits changed during the trial (from 80 to $120 \mathrm{~km} / \mathrm{h}$ ) and each limit was told to each participant by the experimenter at the same point of the route. Finally, the participants filled out the NASA-TLX questionnaire for the occlusion trial and the intolerance of uncertainty questionnaire [2].

\section{Analysis}

In the distraction testing, dependent variables were:

- Percentage of green in-car glances: in-car glance distances below or at median OD (for any 1x1-meter road point).

- Percentage of red in-car glances: in-car glance distances above 85 th percentile OD (for any $1 \times 1$-meter road point).

- Total and mean duration of in-car glances, as well as the percentage of over-2-second in-car glances (after NHTSA [9] verification criteria, for comparison).

- Median in-car glance distance, that refers to distance traveled during an in-car glance.

- Reduced NASA-TLX (no weighting) for measuring experienced task workload (for each condition).

In-car glance durations were scored in real-time by a script reading the pupil's $\mathrm{x}$ and $\mathrm{y}$ coordinates from the eyetracker, and logged with the location data provided by the driving simulator. The durations were scored following the SAE-J2396 definition [11] with the addition of gaze transition time back to driving scene, in order to enable 
more direct comparability with OD. Each glance was manually inspected from a synchronized video (25 fps) by a data reducer for validity using Noldus Observer XT software. All the trials with inaccuracies were manually scored frame-by-frame from the video material. Perfect automated glance recognition was in 33 out of 96 trials $(34.4 \%)$. In total 38 out of 96 trials were manually scored (39.6\%). The automated glance scoring made some false positive in-car glances (mainly glances on the side mirror and the speedometer), but those were manually removed from the data (in $26.0 \%$ of trials). Blinks were removed from the data by rejecting glances shorter than $300 \mathrm{~ms}$.

One-sample sign test was used to test the equality of median green and red in-car glance durations on the two design alternatives (maneuver box locations) to the set verification thresholds $(68 \%$ and $6 \%)$. The differences between the maneuver box locations in the percentages of green and red glances as well as in over-2-second in-car glances were also tested with the sign test due to nonnormal and asymmetric distributions. In addition, paired samples t-test was used for analyzing differences in mean in-car glance durations and total in-car glance durations between the two maneuver box design alternatives. Oneway repeated measures ANOVA was used to test differences in the experienced task workload between the route guidance trials (maneuver box up, down) and the occlusion trial (highway only). Greenhouse-Geisser correction was applied when the sphericity assumption was violated. Bonferroni correction was applied for pairwise comparisons. Partial eta-squared and Cohen's d are reported as metrics of effect size where applicable.

In the occlusion experiment, dependent variables were median occlusion time (OT) and distance (OD). Median was used instead of mean because of the non-gaussian distributions of the occlusion metrics. Only occlusions made on the highway when the speed was over $20 \mathrm{~m} / \mathrm{s}(72$ $\mathrm{km} / \mathrm{h}$ ) were included in order to control for the effects of accelerations and decelerations in the start, junctions, and the end of the trial. Pearson product-moment correlation coefficient was used to test the correlation between median occlusion distance and median in-car glance distance, as well as the correlation between median occlusion distance and intolerance of uncertainty, driving experience, and age.

\section{RESULTS}

\section{Distraction testing - Verification}

Due to low number of in-car glances on the highway routes $(M=8, S D=5$, only one turn per route), it is more reliable to use the suburban routes (up: $M=34$, down: $M=39$, five turns per route) for the verification testing. Medians for the percentage of green glances were up: $67.9 \%$ and down: $60.5 \%$ (Figure 4). The verification threshold of green glances was set to $68 \%$. One-sample sign test indicated no significant differences from 68\% ("median equals to $68 \%$ ", $N=24$ ) for either condition: $p>.999$ (up) and $p=.307$ (down).

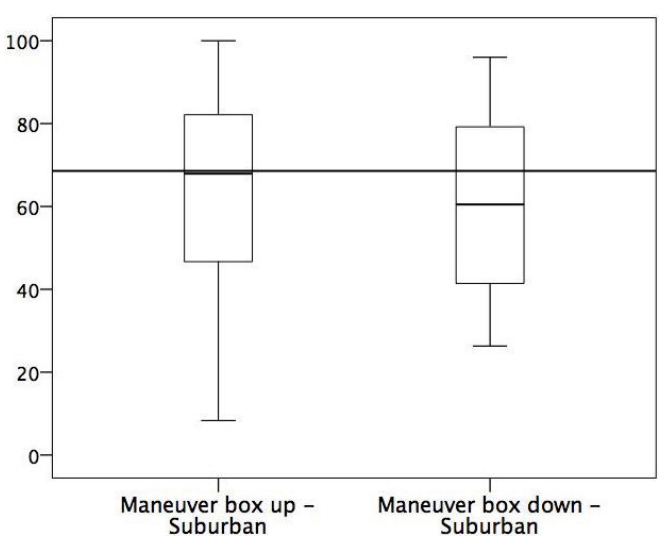

Figure 4: The percentage of green in-car glances. Verification threshold illustrated at $68 \%$ (median should be at or above).

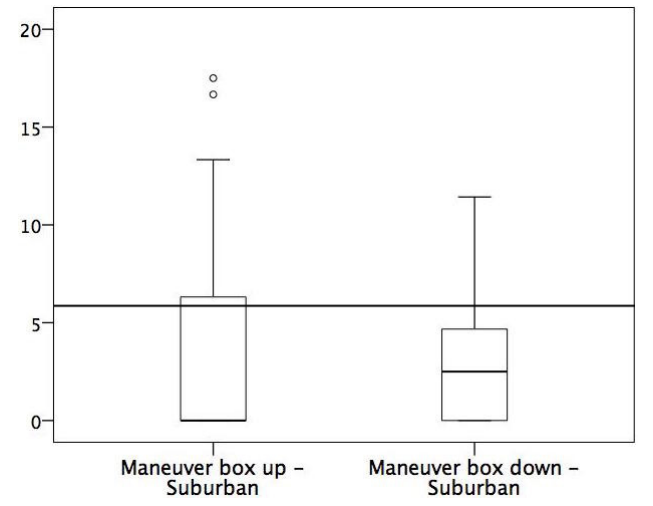

Figure 5: The percentage of red in-car glances. Verification threshold illustrated at $6 \%$ (median should be at or below).

The percentages of red glances were very low in general. Medians for the percentage of red glances were $0.0 \%$ for the up-condition and $2.5 \%$ for the down-condition (Figure $5)$. The verification threshold was set to $6 \%$. One-sample sign test indicated the percentage of red glances was significantly lower from $6 \%$ for the maneuver box down condition, $p<.001$ ("median equals to $6 \%$ ", $N=24$ ). For the maneuver box up condition the difference from $6 \%$ was not significant, $p=.152$. However, note that also the upcondition passed the test, as the median percentage was not significantly higher than $6 \%$ (median for up was $0.0 \%$ ).

\section{Maneuver box location}

We did not find significant effects of the maneuver box location on the metrics of [6], see Figures 4 and 5 (green glances: $p>.999$, red glances: $p=.383, N=24$ ). For comparison, we also wanted to see if there were significant differences between the two alternative designs with the metrics of NHTSA [9] (for the suburban routes). However, note that the NHTSA metrics are not directly applicable here due to the dynamic and self-paced driving scenarios.

No significant effects of the maneuver box location on total in-car glance durations were found ( $p=.153, N=24)$. Notable are the long total in-car glance durations for the tasks (up: $M=27.6 \mathrm{~s}, S D=11.3$; down: $M=31.6 \mathrm{~s}, S D=$ 17.2), well exceeding the NHTSA [9] recommendation of 
$12 \mathrm{~s}$ (max). However, this metric is directly dependent on the experimental design; on how many turns there are to make until reaching the destination, and not applicable here. Mean in-car glance durations stayed well below 1 second (up: $M=.82 \mathrm{~s}, S D=.12$; down: $M=.82 \mathrm{~s}, S D=.16$ ). There was no significant effect of the maneuver box location on mean in-car glance durations $(p=.839, N=24)$. The percentage of over-2-second in-car glances was very low (up: $M=0.35 \%, S D=.96$; down: $M=0.29 \%, S D=1.41$ ), further indicating low visual demands of the route following tasks. There was no significant effect of the maneuver box location on the percentage of over-2-second in-car glances $(p>.999, N=24)$.

\section{Experienced workload: NASA-TLX}

There were no significant effects of the maneuver box location on the experienced workload on highway driving (Figure 6, mean difference down-up: .07, $p=.980$ ). However, the occlusion trial was experienced significantly more demanding than the route guidance trials $\left(F(1.57,36.18)=53.70, p<.001\right.$, partial $\left.\eta^{2}=.700\right)$. There were significant differences on the experienced workload between the occlusion trial and the maneuver box up trial (mean difference: 31.11, $p<.001$ ), and between the occlusion trial and the maneuver box down trial (mean difference: $31.04, p<.001$ ). The experienced workload was at a highly similar level across the highway and suburban routes in the distraction testing (up: $p=.697$, down: $p=$ $.831)$.

\section{Comparability across routes with different visual demands}

When comparing the suburban test results with the highway test results, we found the same insignificant relative effects of the maneuver box location, similar mean in-car glance durations (up: $.82 \mathrm{~s}$, down: $.85 \mathrm{~s}$ ) and similar very low percentages of over-2-second in-car glances $(\sim 0 \%)$. Yet the percentages of red glances were higher (median up: $4.5 \%$, median down: $15.5 \%$ ) and the percentages of green glances were lower (median up: $38.8 \%$, median down: $41.7 \%$ ) than on the suburban routes. However, the highway data can be considered as unreliable due to low number of in-car glances ( $M=8$ glances for both conditions).

Occlusion experiment - Occlusion times and distances The distributions of the drivers' median occlusion times (OT, Figure 7) and distances (OD, Figure 8) on the highway were fairly similar with the distributions reported in [7], with a slight skew towards the lower ODs. Median OD ranged from 6.2 to $50.4 \mathrm{~m}$.

\section{Occlusion distance vs. in-car glance distance}

In-car glance distance refers to the distance that is traveled during an in-car glance. Median in-car glance distances correlated significantly between the suburban up and down trials $(r=.633, p=.001)$ and were averaged for data reduction and comparison with median ODs. We found significant correlation between the drivers' median OD and median in-car glance distance, $r=.47, p=.020$ (Figure 9).

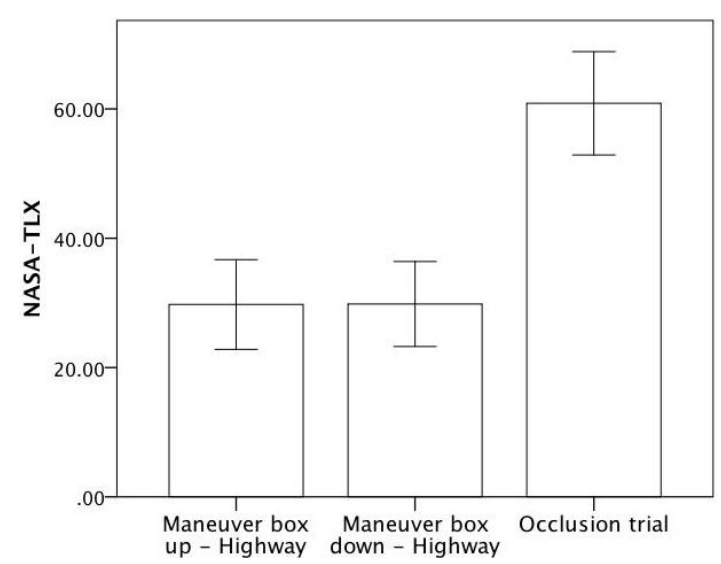

Figure 6: Total workload - highway, NASA-TLX (max. 100). Error bars: $95 \%$ CI.

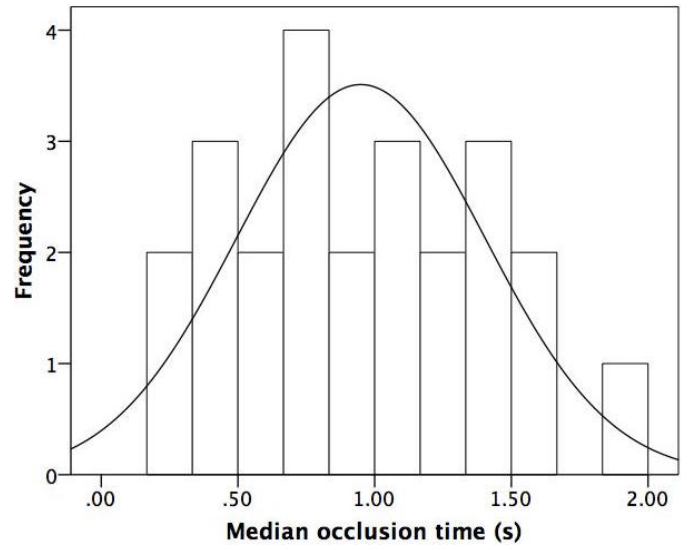

Figure 7: Median occlusion times (s) on highway (speed $>72$ $\mathbf{k m} / \mathbf{h})$.

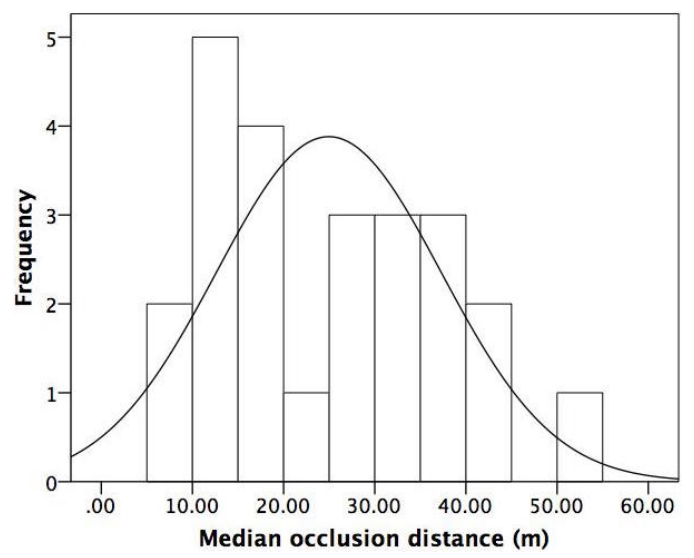

Figure 8: Median occlusion distances (m) on highway (speed > $72 \mathrm{~km} / \mathrm{h})$.

\section{Occlusion distance vs. intolerance of uncertainty}

We found no correlation between median occlusion distances and intolerance of uncertainty $(r=.034, p=$ .873). However, there were significant inverse correlations between age and median OD $(r=-.653, p=.001$, Figure $10)$ as well as between driving experience and OD $(r=-$ .637, $p=.001)$. Here, driving experience correlated strongly with age $(r=.993, p<.001)$. 


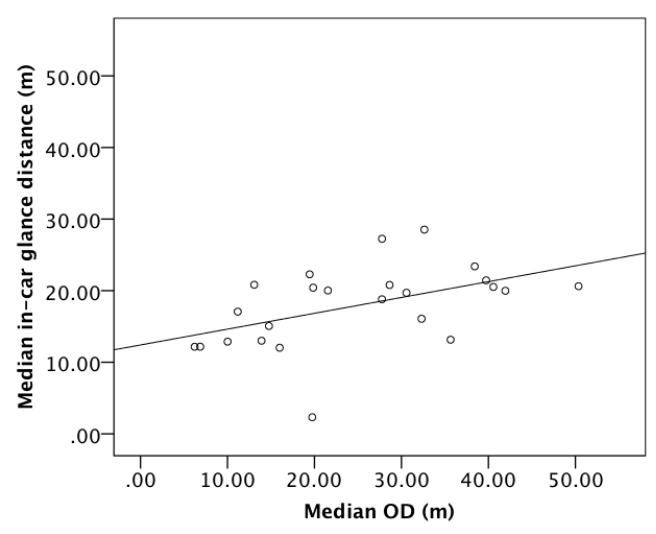

Figure 9: Median in-car glance distance $(m)$ on the suburban routes vs. median OD (m) on highway.

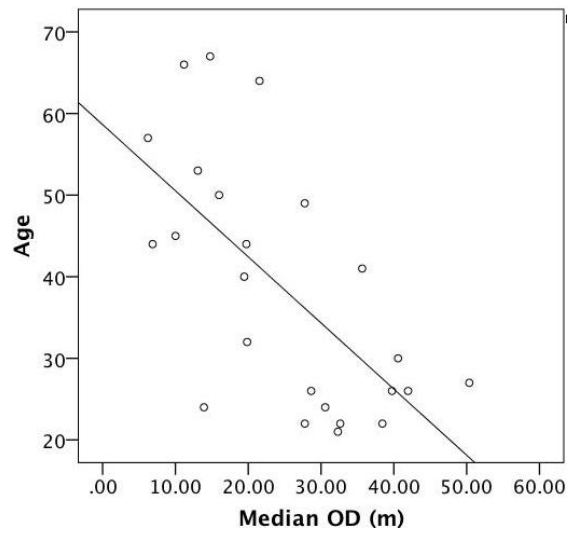

Figure 10: Age vs. median OD (m, highway).

\section{GENERAL DISCUSSION}

We studied the visual distraction effects of audio-visual route guidance in ecologically realistic driving scenarios with dynamic maneuvers and self-controlled speed. In the distraction testing part of the study we studied a commercial audio-visual route guidance system prototype following the testing and verification criteria described in [6]. The testing method enabled tactical control of multitasking for the participants, but in controlled settings.

Based on the percentages of red and green glances, the route guidance user interfaces under testing passed the set verification criteria. The audio-visual route guidance aids passed the test even if glances on the in-car display were required mostly on the visually high-demanding parts of the routes (i.e., before and at the turns). The current data can act as a baseline for an acceptable in-car task to which more complex in-car tasks, involving also visual-manual interactions, can be benchmarked against.

There were no significant effects of the maneuver box location (up, down) on the in-car display on any metric. In addition, there were no significant differences on the experienced workload between the two different maneuver box locations. The possible effects of showing more upcoming maneuvers above the maneuver box (down) should be studied.
We found differences in the verification metrics between the suburban and highway scenarios, but the highway test results can be considered unreliable due to low number of in-car glances (highway routes involved only one turn per route vs. five turns on the suburban routes). This finding suggests that a representative number of in-car glances should be collected per in-car task in order to get reliable verification data. For short tasks, the tasks should be repeated so that at least 20 in-car glances can be examined for red and green glances. In this way, single red in-car glances do not distort the percentages. There are definitely more complex road environments to navigate than the ones in the study but many of the participants took wrong turns (trials excluded in the results), which could suggest the route finding task itself was fairly difficult. However, the effects of the test route on the verification test results should be more carefully studied in following research.

The second part of the study was focused on method validation by the means of visual occlusion. The distributions of the drivers' median occlusion times and distances on the highway were fairly similar with the distributions reported in [6], and thus suggesting a representative sample of the driver population. This kind of sample validation must be an integral part of the verification testing, and is missing from, for instance, in the current NHTSA [9] test guidelines. It seems the NHTSA verification results are highly dependent on the distribution of 'short-glancers' and 'long-glancers' in the sample [1]. This finding is understandable given the large variance in the preferred occlusion times and distances the drivers are willing to tolerate (see Figures 9 and 10, see also [7] and [8]).

We found a significant correlation between the drivers' median occlusion distance and median in-car glance distance. This finding gives support for the testing method [6]; the idea that the drivers' self-preferred occlusion distances can be used as a comparison point for appropriate visual in-car glancing behavior - and thus, visual distraction. However, this finding should be replicated with other types of in-car tasks. Moreover, the correlation tells us there are differences in either drivers' uncertainty (or risk) tolerance levels, or that other drivers are just more apt to drive longer distances essentially blind than others. In the latter case, it would be fairer to compare each driver's incar glance distances to his or her individual preferred ODs. However, like discussed in [6], the high-OD drivers in [7] were associated with decreased lane-keeping performance, suggesting overestimation of their visual sampling skills.

The occlusion experiment represents a baseline comparison point for a driving scenario with a 'maximum level of tolerated visual inattention' while focusing on driving only. NASA-TLX for highway driving indicated that the occlusion trial was experienced significantly more demanding than the route guidance trials. This is in line with the test data, suggesting the demands of the route 
guidance following were at a low level. When it comes to the intolerance of uncertainty [2], we found no correlation between drivers' median occlusion distances and intolerance of uncertainty. This suggests that the general intolerance of uncertainty - a personality trait - is not one of the factors behind the individual differences on the preferred occlusion distances or on the in-car glance distances. Instead, both age as well as driving experience had significant inverse correlations with median occlusion distance. These are related variables, and the exact factors behind the preferred occlusion distances are a topic for further research. These could be, for instance, the spatial span of working memory [5], that has been observed to decrease with age, or some skill acquired with increasing driving experience. However, the study of [7] with a larger sample did not indicate significant effects of driving experience on OD. In the study, neither did age correlate significantly with OD $(p=.090)$, but the sample was not equally distributed across different age groups. However, in order to have a comparable distribution of 'short-glancers' and 'long-glancers' in a test group, the NHTSA [9] guidelines on the age distribution of the drivers can be recommended.

Finally, it should be noted that the testing method applies only to driving scenarios with empty roads [6]. The road environment-based ODs are not a reliable baseline if there is other traffic on the roads, as the traffic will likely increase the visual demands of driving. Future research should address how one could define baseline ODs (or OTs) for even more dynamic traffic scenarios including other traffic.

\section{CONCLUSION}

This was the first controlled quantitative analysis on the visual distraction effects of audio-visual route guidance in simulated, but ecologically realistic driving scenarios with dynamic maneuvers and self-controlled speed. The results suggest that the visual distraction effects of audio-visual route guidance are low. The findings provide general support for the testing method, which uses drivers' preferred occlusion distances on the selected test routes as the baseline for acceptable in-car glance durations.

\section{ACKNOWLEDGMENTS}

The study was funded by HERE Global B.V.

\section{REFERENCES}

1. Mikael L. Aust, Annie Rydström, Robert Broström, and Trent Victor. 2015. Better ways to calculate pass/fail criteria for the eye glance measurement using driving simulator test. In Proc. of the $4^{\text {th }}$ International Conference on Driver Distraction and Inattention.

2. Jane Birrell, Kevin Meares, Andrew Wilkinson and Mark Freeston. 2011. Toward a definition of intolerance of uncertainty: A review of factor analytical studies of the Intolerance of Uncertainty Scale. Clinical Psychology Review, 31, 7, 1198-1208. http://dx.doi.org/10.1016/j.cpr.2011.07.009
3. Robert Broström, Mikael L. Aust, Linnea Wahlberg and Laban Källgren. 2013. What drives off-road glance durations during multitasking-capacity, practice or strategy. In Proc. of the 3rd International Conference on Driver Distraction and Inattention, 4-6.

4. Sandra G. Hart and Lowell E. Staveland. 1988. Development of NASA-TLX: Results of empirical and theoretical research. In Human Mental Workload, Peter A. Hancock and Najmedin Ed Meshkati (eds.). Elsevier, Amsterdam, 139-183.

5. Lisa Jenkins, Joel Myerson, Jennifer A. Joerding and Sandra Hale. 2000. Converging evidence that visuospatial cognition is more age-sensitive than verbal cognition. Psychology and Aging. 15, 1, 157-175. http://dx.doi.org/10.1037/0882-7974.15.1.157

6. Tuomo Kujala and Jakke Mäkelä. 2015. Development of a testing environment and a verification procedure for in-car tasks with dynamic driving scenarios. In Proc. of the $4^{\text {th }}$ International Conference on Driver Distraction and Inattention.

7. Tuomo Kujala, Jakke Mäkelä, Ilkka Kotilainen and Timo Tokkonen. 2016. The Attentional Demand of Automobile Driving Revisited: Occlusion Distance as a Function of Task-Relevant Event Density in Realistic Driving Scenarios. Human Factors, 58, 1, 163-180. http://dx.doi.org/10.1177/0018720815595901

8. Ja Young Lee, Madeleine Gibson and John D. Lee 2015. Secondary task boundaries influence drivers' glance durations. In Proc. of the 7th International Conference on Automotive User Interfaces and Interactive Vehicular Applications, 273-280. http://dx.doi.org/10.1145/2799250.2799269

9. National Highway Traffic Safety Administration. 2013. Visual-manual NHTSA driver distraction guidelines for in-vehicle electronic devices (NHTSA-2010-0053). Washington, DC: NHTSA.

10. John W. Senders, A. B. Kristofferson, W. H. Levison, C. W. Dietrich and J. L. Ward. 1967. The attentional demand of automobile driving. Highway Research Record, 195.

11. Society of Automotive Engineers. 2000. SAE-J2396 Definitions and Experimental Measures Related to the Specification of Driver Visual Behavior Using Video Based Techniques. Warrendale, PA: SAE.

12. Louis Tijerina, S. Johnston, E. Parmer, M. D. Winterbottom and M. Goodman. 2000. Driver distraction with wireless telecommunications and route guidance systems (No. HS-809 069). Washington, DC: NHTSA.

13. Omer Tsimhoni and Paul Green. 1999. Visual demand of driving curves determined by visual occlusion. In Proc. of Vision in Vehicles 8 Conference. 